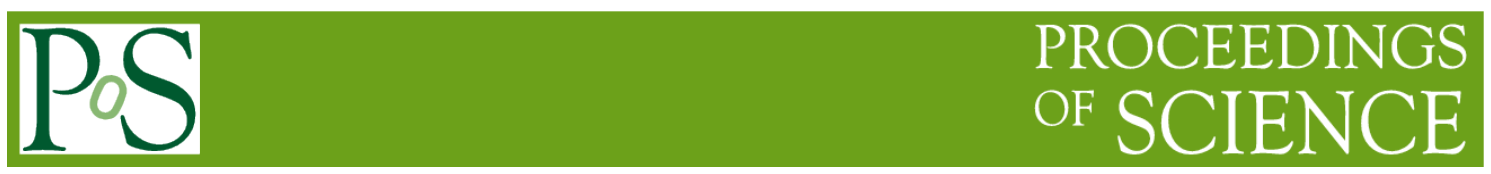

\title{
Highlights of LHC experiments - Part I
}

\section{Dave Charlton ${ }^{1}$}

University of Birmingham

Birmingham B15 2TT, United Kingdom

E-mail: dave. charltondcern.ch

The superb performance of the LHC accelerator in 2016, in both live time and peak luminosity, has provided a large data sample of collisions at $13 \mathrm{TeV}$. Excellent performances of the ATLAS and $\mathrm{LHCb}$ detectors, together with highly performant offline and analysis systems, mean that a wealth of results are already available from $13 \mathrm{TeV}$ data. Selected highlights are reported here.

38th International Conference on High Energy Physics

3-10 August 2016

Chicago, USA

\footnotetext{
${ }^{1}$ Speaker, on behalf of the ATLAS and LHCb Collaborations
} 


\section{Introduction}

The first multi-year data-taking run of the Large Hadron Collider, LHC, at CERN was marked for a wealth of physics results, including the discovery of the Higgs boson by ATLAS and CMS in 2012 [1]. Those data, collected in 2009-2013, formed the "Run-1" dataset. Datataking resumed at $13 \mathrm{TeV}$ proton-proton centre-of-mass energy $\left(ل_{s}\right)$ in 2015, providing around 4 $\mathrm{fb}^{-1}$ to ATLAS and CMS, together with a $\mathrm{Pb}-\mathrm{Pb}$ heavy-ion run. Proton-proton data-taking in 2016 [2] has seen a substantially increased LHC live fraction, higher peak luminosities (up to $1.2 \times 10^{34} \mathrm{~cm}^{-2} \mathrm{~s}^{-1}$ to ATLAS and CMS by the time of this conference), and up to 2200 colliding bunches in the accelerator. A data sample of more than $21 \mathrm{fb}^{-1}$ has been recorded in Run- 2 by the time of the conference by ATLAS, and $1.2 \mathrm{fb}^{-1}$ by LHCb. ATLAS reported results using 3-15 $\mathrm{fb}^{-1}$ of Run-2 pp data, as well as Run-1 measurements (not covered here). LHCb results reported here use Run-1, or the 2015 Run-2, data.

The detector configuration of $\mathrm{LHCb}$ is essentially unchanged from that of Run-1 [3], the main new component being the installation of scintillators ("Herschel") along the beam directions up to $114 \mathrm{~m}$ away from the LHCb interaction point. Major changes have been made to the trigger architecture, in addition, so that now part of the high-level trigger runs asynchronously from disk buffers after automated calibration and alignment. This results in analysis-quality physics data being available directly from the detector.

Relative to the detector configuration of Run-1 [4], ATLAS has installed a new innermost layer of pixel sensors for Run-2, providing a four barrel-layer pixel detector. The innermost layer of sensors lies at $3.4 \mathrm{~cm}$ radially from the interaction region. Furthermore, one arm of a new forward detector system ("AFP") has been installed in Roman Pots on the outgoing proton beam in one direction. The second arm will be installed in early 2017. Further substantial work has been done on the readout of many systems, and on the high-level trigger, to allow operation at up to $100 \mathrm{kHz}$ first-level trigger rate, and $1 \mathrm{kHz}$ output rate (compared to $200-400 \mathrm{~Hz}$ in Run1), even with the high instantaneous luminosities of Run-2 which have resulted in "pile-up" (multiple $p p$ interactions per bunch-crossing) up to mean values of 40 at the starts of fills, with the average value during a fill close to the design peak value of 23 interactions per crossing.

Maintaining consistently excellent performance of the ATLAS trigger system [5] is a significant operational challenge, especially as the luminosity and pileup rises. The power of the first and high-level trigger systems of ATLAS is illustrated by the fact that it has been possible to maintain the use of inclusive single-lepton ( $e$ and $\mu$ ) triggers with transverse momentum $\left(p_{\mathrm{T}}\right)$ thresholds of 24-26 GeV, the inclusive missing transverse momentum $\left(E_{\mathrm{T}}{ }^{\text {miss }}\right)$ trigger threshold around $100 \mathrm{GeV}$, and multi-object trigger thresholds also rather low, for example a diphoton trigger, crucial for the $H \rightarrow \gamma \gamma$ channel, with thresholds of 35 and $25 \mathrm{GeV}$ on the leading and subleading photons, respectively. Example trigger efficiency curves, relative to offline selections, are illustrated in Figure 1. 

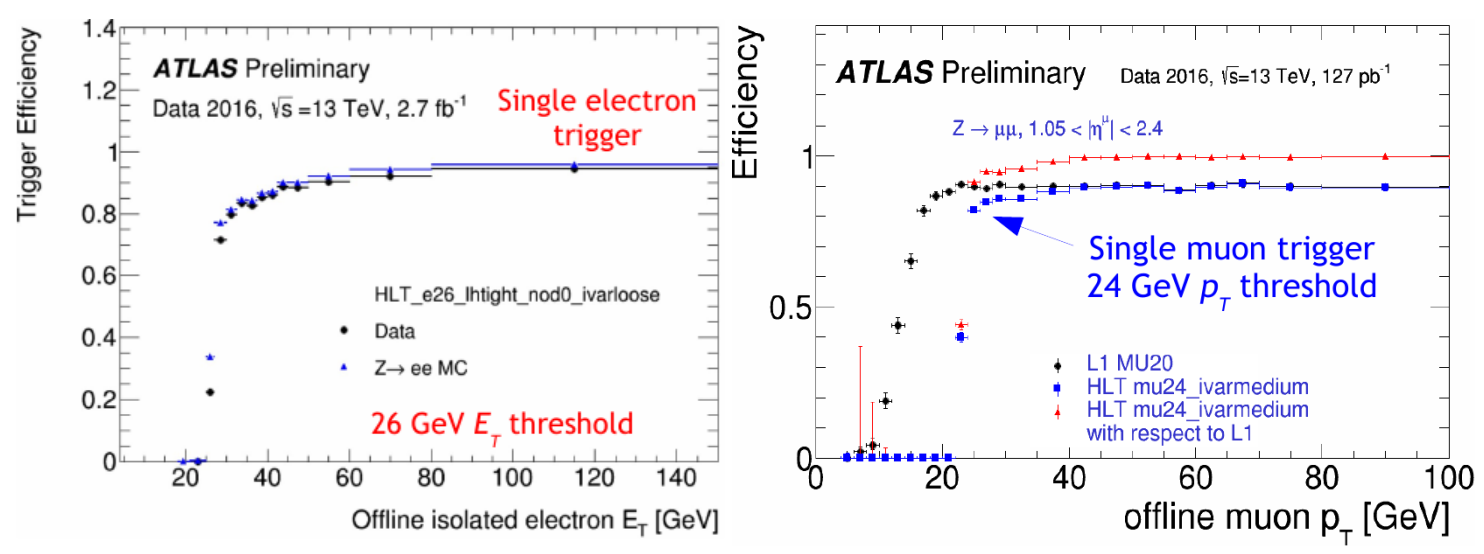

Figure 1: ATLAS electron (left) and muon (right) trigger efficiencies with respect to offline selections.

\section{Measurements}

Many measurements have been released with Run-1 and Run-2 data, across all accessible sectors of the Standard Model. The huge data samples, together with the excellent performance of the detectors, mean both that benchmark final-states can be measured with great precision, and that increasingly complex final-states can be probed. This programme of work is complemented by the exceptional progress in Monte Carlo modelling: next-to-leading order (NLO, in $\alpha_{\mathrm{s}}$ ) event generators are now standard tools, and NNLO calculations, even NNNLO in some cases, are becoming increasingly available for a range of processes. Such progress is fundamentally important to extract the most from the LHC data, and to help map data-driven background predictions solidly into signal regions, when searching for new physics signatures in complex event topologies.
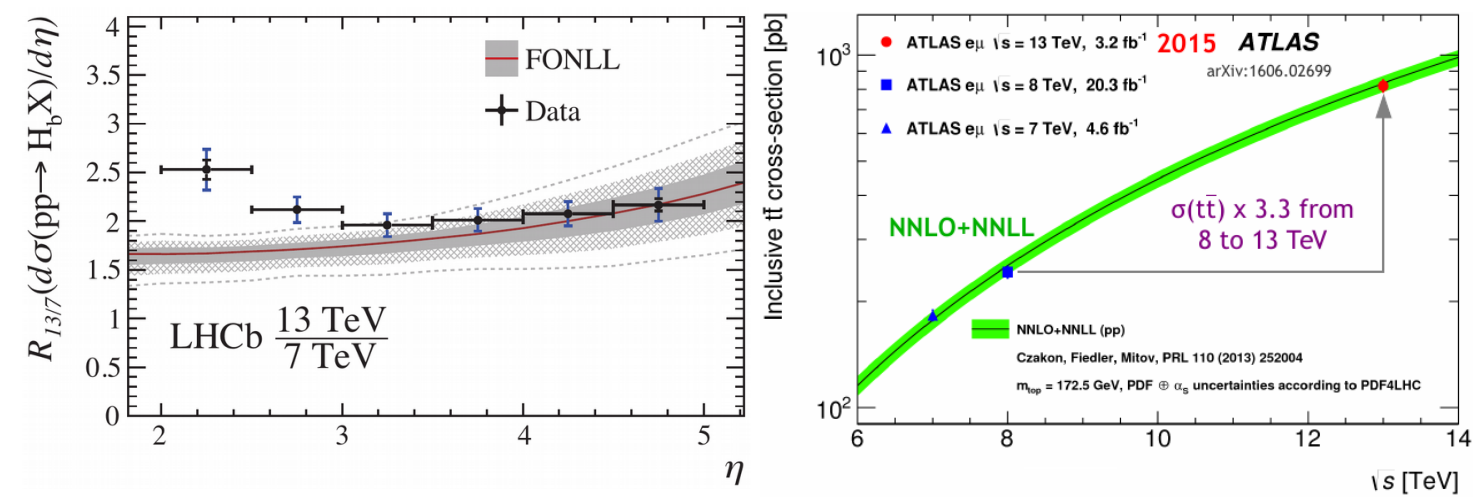

Figure 2: (Left) Ratio of $b \bar{b}$ cross-section measurements within the LHCb acceptance between 13 and $7 \mathrm{TeV}$ [6]; (Right) Inclusive $\bar{t} t$ cross-section as a function of $\sqrt{\mathrm{s}}_{\text {[7] }}$.

Examples of cross-section measurements in the heavy quark sector are shown in Figure 2: the ratio of the $b \bar{b}$ cross-section measured in the range $2<\eta<5$ by LHCb at 7 and $13 \mathrm{TeV}$ centre-of-mass energies [6] is shown in the left pane, that from ATLAS of the inclusive $\bar{t} t$ crosssection [7] on the right. The $b \bar{b}$ cross-section measurement from LHCb uses semileptonic $b$ hadron decays, and the ratio of cross-sections between the two centre-of-mass energies is relatively poorly described by the FONLL [8] predictions for the lower $\eta$ values measured. For 
the inclusive $t \bar{t}$ cross-section measurement, the NNLO+NNLL predictions (see Ref. [7] for a discussion of the calculations) continue to provide an excellent description also at $13 \mathrm{TeV}-$ but the theoretical precision, estimated to be $5 \%$, is bettered by the experimental one of $3.9-4.4 \%$. Detailed studies of top-pair production properties have also been reported [9].

A range of measurements have been reported in the electroweak sector. Two examples are detailed studies of the properties of $Z+$ jets events [10], and the production of a pair of electroweak vector bosons, for example $W Z$ [11]. These are illustrated in Figure 3.
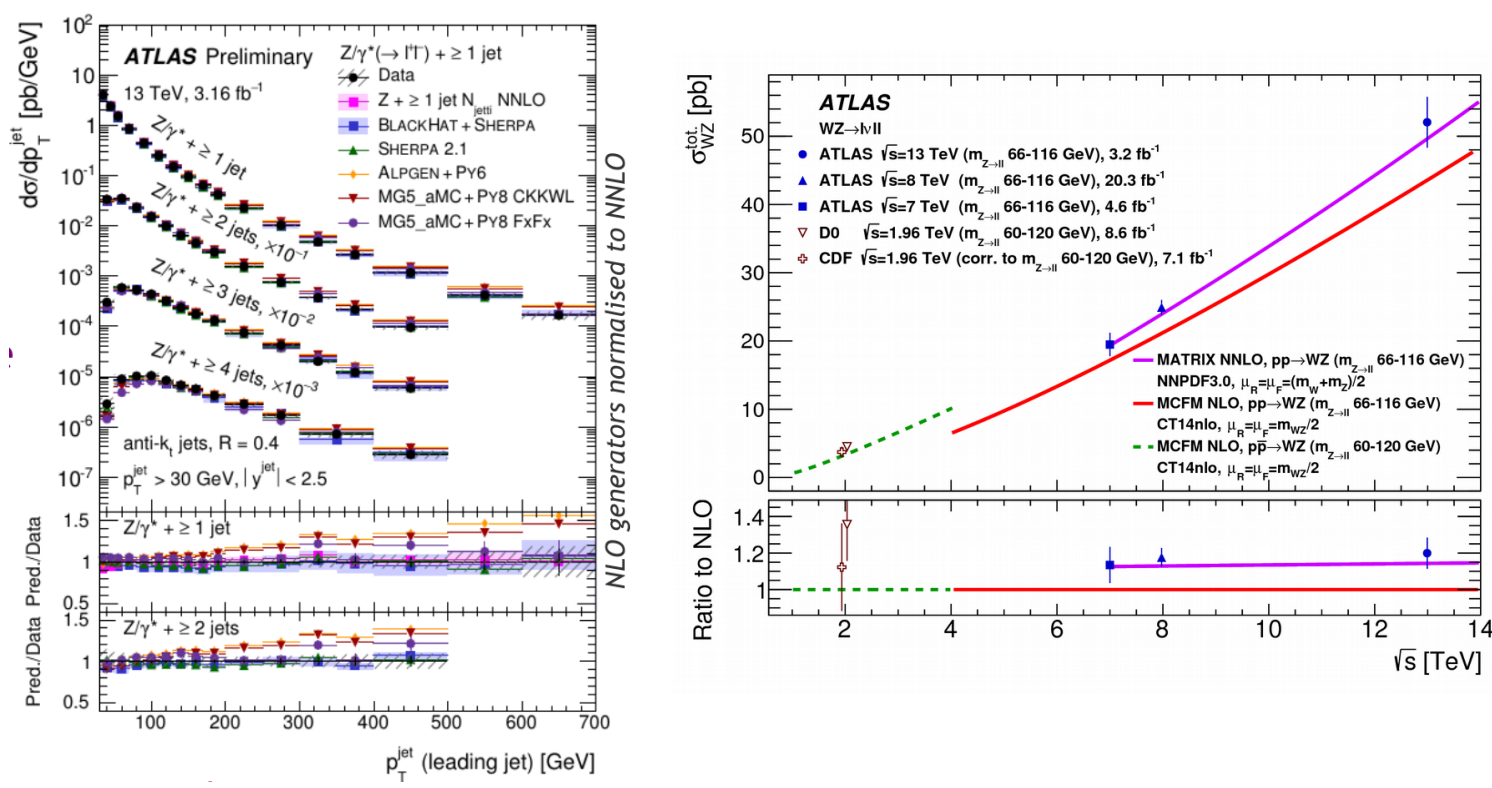

Figure 3: (Left) Fully corrected spectrum of leading jet $p_{\mathrm{T}}$ in $Z+$ jets events, with different jet multiplicity thresholds [10]; (Right) Inclusive $W Z$ cross-section as a function of $\sqrt{s}_{s}$ measured using $W Z \rightarrow \ell v \ell \ell$ decays[11].

In the $\mathrm{Z}+$ jets case, the NLO predictions (labelled BLACKHAT+SHERPA and SHERPA 2.1 in the figure) do a good job of describing the shape of the spectrum in the data, and are aligned with the NNLO prediction for $Z+\geq 1$ jet production; the leading-order Monte Carlos ALPGEN and MadGraph5, interfaced with PYTHIA, over-predict the relative yield of high- $p_{\mathrm{T}}$ jets. The interested reader is referred to Ref. [10] for a detailed discussion of the calculations entering the different predictions, and their references. In the case of the inclusive $W Z$ cross-section, it is striking that the data - measured with $7 \%$ precision at $13 \mathrm{TeV}$ - are well-described by the NNLO predictions [12] which lie around $20 \%$ above the NLO calculations. This apparently resolves a puzzle remaining from Run-1 as to why the diboson cross-sections were not well described by the earlier Standard Model calculations.

\section{CP Violation at $\mathrm{LHCb}$}

Among the measurements of $\mathrm{CP}$ violation reported at this conference by $\mathrm{LHCb}$, two new results are flagged here: the first evidence for $\mathrm{CP}$ violation in a $b$-baryon system [13], and a significantly improved measurement of potential CP violation in the charm sector [14]. The $b$ baryon measurement uses $\Lambda_{b} \rightarrow p \pi^{-} \pi^{+} \pi^{-}$decays, using the opening angle $\Phi$ between the $p \pi^{-}$fast and $\pi^{+} \pi_{\text {slow }}^{-}$decay planes. The asymmetry constructed as a function of this angle is shown in Figure 4 - the consistency of these measurements with a constant value of zero is at the $3.3 \sigma$ 
level. $\mathrm{LHCb}$ interpret this as first evidence for $\mathrm{CP}$ violation in the baryon sector. In the charm sector, LHCb have a new measurement of $A_{\mathrm{CP}}\left(K^{+} K^{-}\right)$which tags the $D^{0}$ flavour from that of the slow pion in $D^{*+} \rightarrow D^{0}\left(K^{+} K^{-}\right) \pi^{+}$decays. Combining this with a previous measurement using a semileptonic muon charge tag yields [14]: $A_{\mathrm{CP}}\left(K^{+} K^{-}\right)=(0.04 \pm 0.12$ (stat) \pm 0.10 (syst) $) \%$, the most precise result from an individual experiment. Many more results from LHCb are reported in another plenary contribution [15].
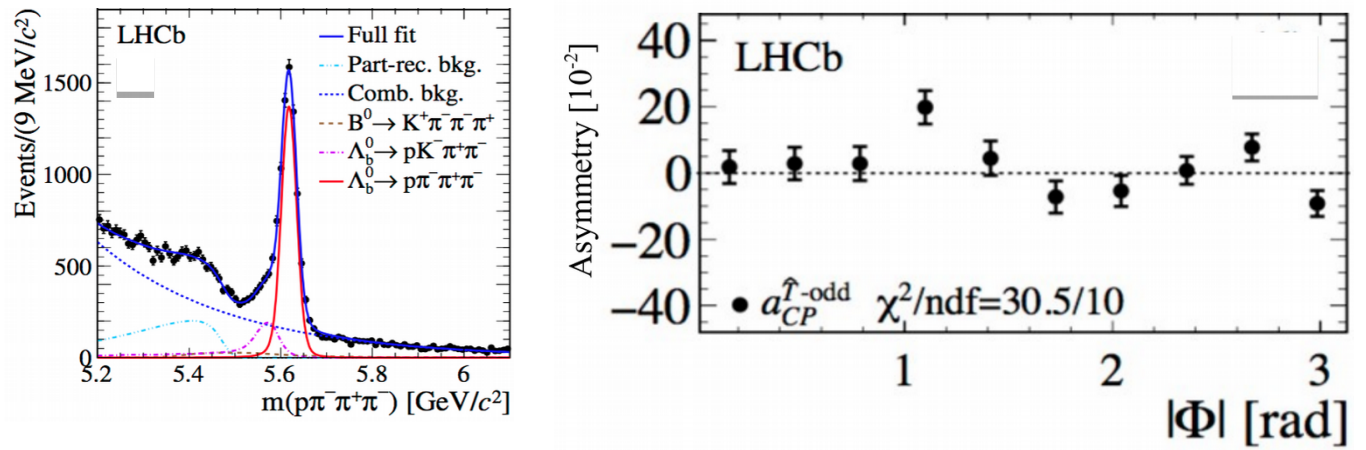

Figure 4: Evidence for CP violation in the $b$-baryon sector: (left) reconstructed mass distribution of $p \pi^{-} \pi^{+} \pi^{-}$decay candidates, showing the clear $\Lambda_{b}$ signal; (right) the asymmetry constructed as a function of the angle $\Phi$.

\section{Elucidating the $125 \mathrm{GeV}$ Higgs Boson}
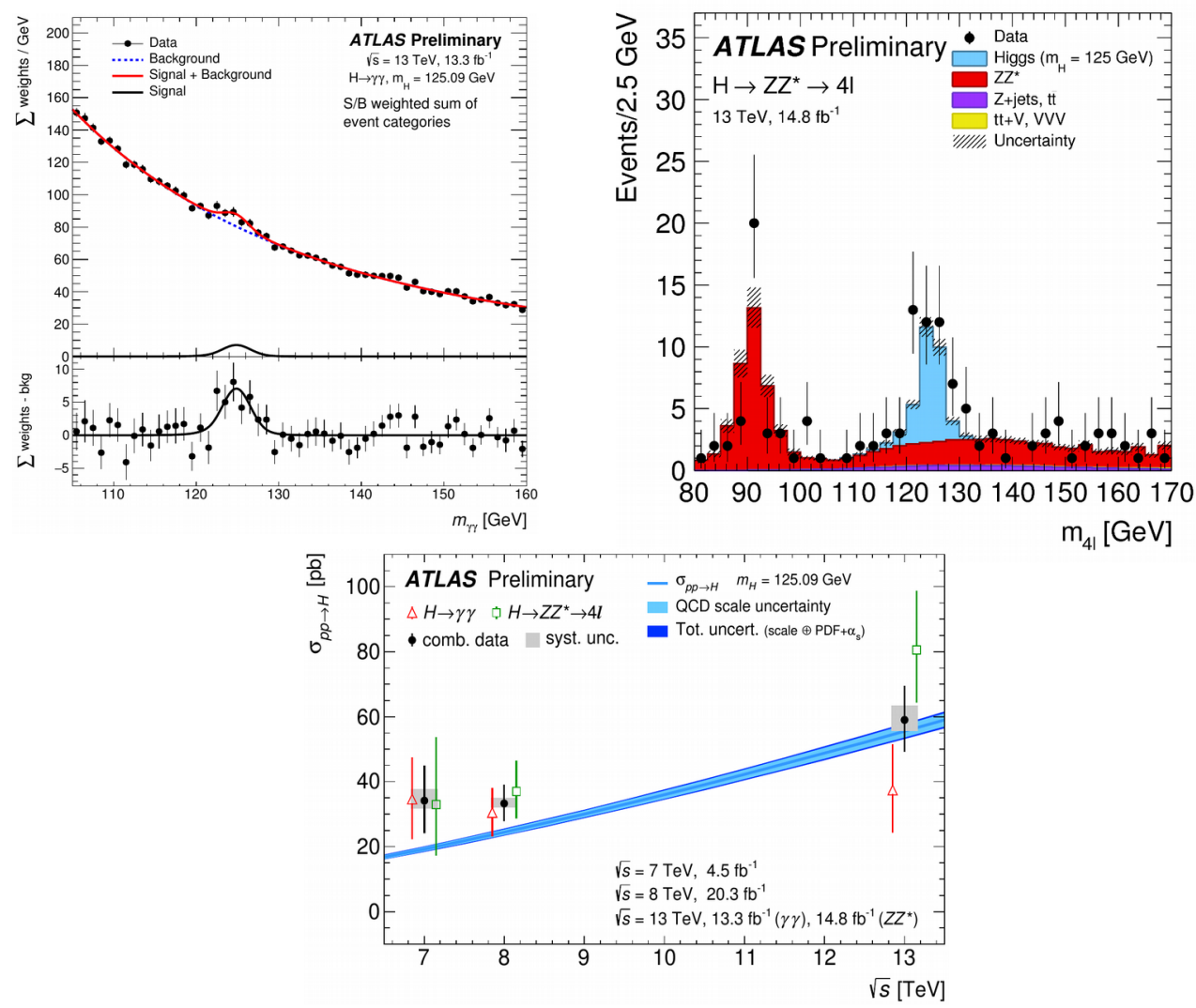

Figure 5: Invariant mass distributions of $H$ candidates in the $H \rightarrow \gamma \gamma$ [16] and $H \rightarrow 4 \ell$ [17] channels (top left and right, respectively), and combined measurement of the $H$ production cross-section at different centre-of-mass energies [18] (bottom). 
With the large data samples being accumulated at $13 \mathrm{TeV}$, re-establishing the production of the $H(125)$ at the new centre-of-mass energy was an early priority for ATLAS, already achieved by the time of the conference for the $H \rightarrow \gamma \gamma$ [16] and $H \rightarrow 4 \ell$ [17] channels, as shown separately and combined [18] in Figure 5. The combined ATLAS inclusive cross-section measurement for $H$ production at $13 \mathrm{TeV}$ is $\sigma(p p \rightarrow H+X, 13 \mathrm{TeV})=59.0^{+9.7}{ }_{-9.2}(\text { stat })^{+4.4}{ }_{-3.5}($ syst $)$ $\mathrm{pb}$, consistent with the Standard Model prediction [19] of 55.5 $5_{-3.4}^{+2.4} \mathrm{pb}$. The Run-2 data are used also to make differential cross-section measurements [16]. The next big steps for the $H(125)$ measurements, and thus key goals for Run-2, include establishing the $\bar{t} H$ associated production process to probe directly the $t t H$ vertex, and observing, with the customary $5 \sigma$ significance, the $H \rightarrow b \bar{b}$ decay mode. These analyses are in progress and results with the data samples available are shown in Figure $6[20,21]$ - more data and study are still needed to establish these processes experimentally.
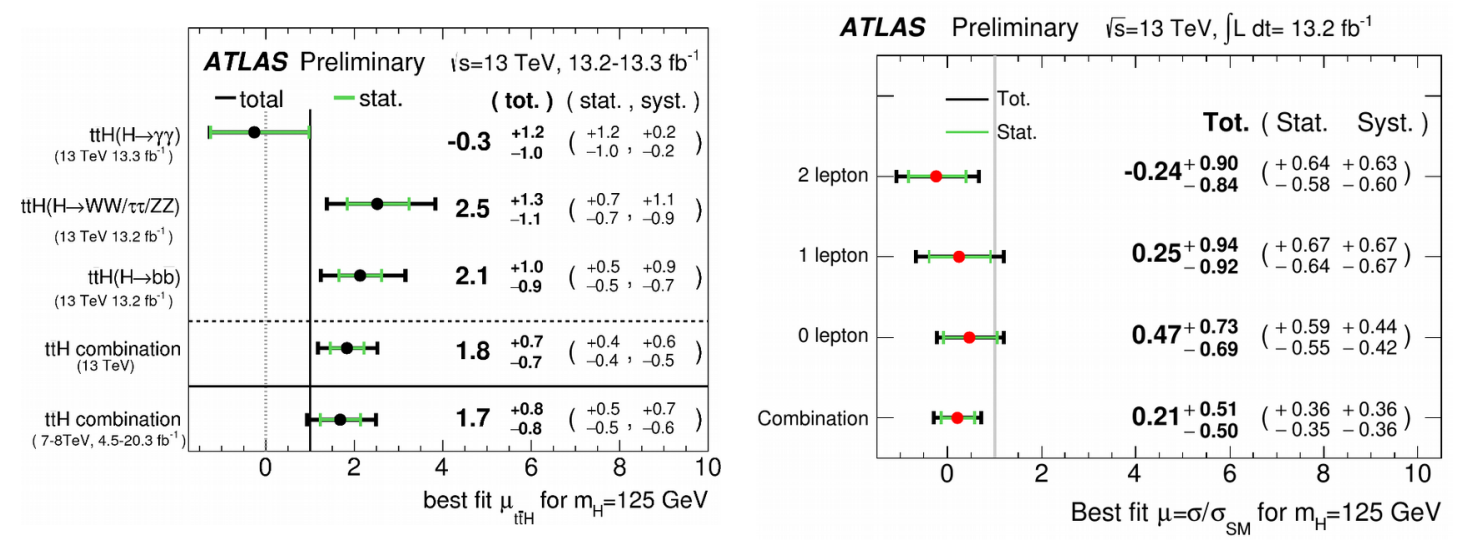

Figure 6: Measured signal strengths, normalised to the Standard Model expectations, for $\bar{t} H$ production [20] (left) and the $H \rightarrow b \bar{b}$ decay [21] (right).

\section{Searches for Physics Beyond the Standard Model}

The sample of around $15 \mathrm{fb}^{-1}$ of $13 \mathrm{TeV} p p$ collision data available at the time of the conference already provides a major increase in sensitivity for searches for new physics at high masses, pushing the reach well beyond that of Run-1, probing into the $\mathrm{TeV}$, even multi-TeV, mass range. ATLAS is probing widely and deeply into many event topologies, looking for possible deviations from Standard Model expectations which could start to signal new particles or new physics effects. A host of search results using the $13 \mathrm{TeV}$ data sample were shown at the conference, and are reported in other talks [22]. In this contribution only a small subset can be covered. All analyses mentioned here are updated to include both 2015 and 2016 data.

One of the simplest signatures, where the Standard Model cross-section is also very high, is to look for new resonances, or non-resonant effects, in dijet production at high mass [23]. The dijet invariant mass distribution for central jet production is shown in Figure 7, along with the angular distributions in the dijet centre-of-mass frame expressed in terms of the $\chi$ variable [23], for different dijet mass intervals. No significant deviations are observed from the Standard Model expectations, and constraints are placed on new physics [23]: for example production of an excited quark $\left(q^{*}\right)$ is excluded at $95 \% \mathrm{CL}$ for a mass below $5.6 \mathrm{TeV}$, to be compared with the Run-1 equivalent lower limit of $4.1 \mathrm{TeV}$; four-quark contact interactions introduced via a simple four-point term in the lagrangian are excluded (at 95\% CL) for scales $\Lambda$ below 12.6 and 19.9 
$\mathrm{TeV}$ for positive and negative signs of couplings - here the equivalent Run-1 lower limits were 8.1 and $12.0 \mathrm{TeV}$, respectively.
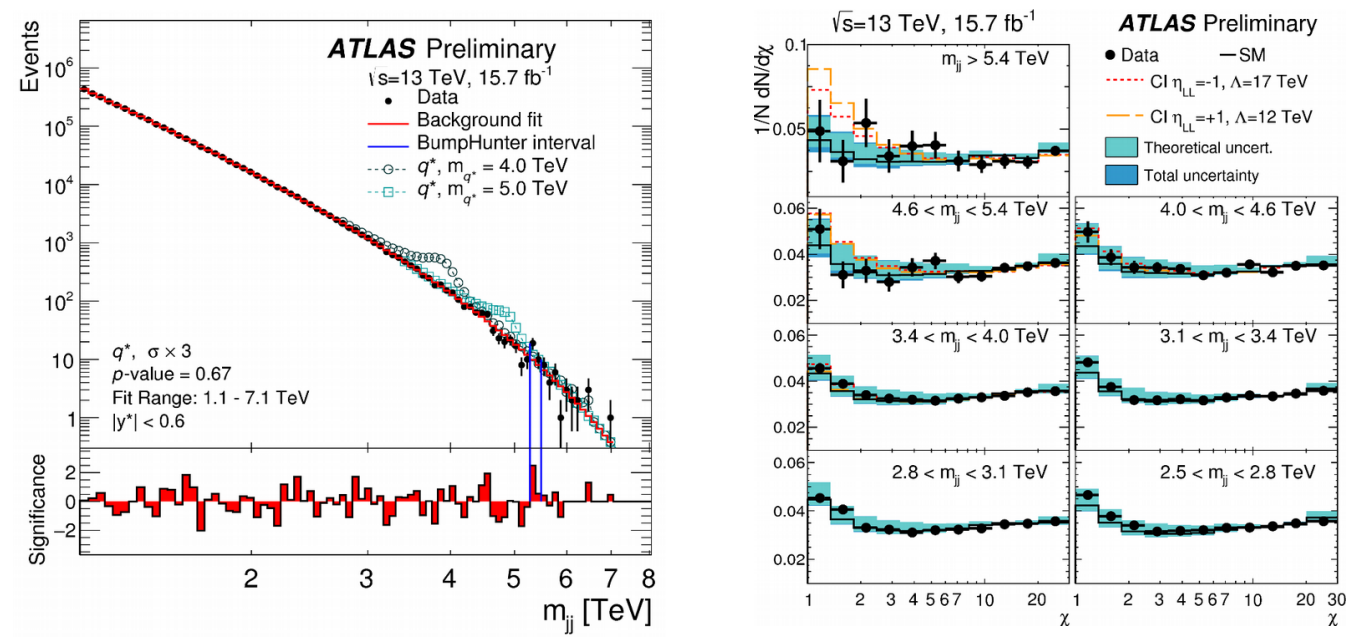

Figure 7: Dijet invariant mass distributions in the high-mass dijet search (left) and angular distributions for different dijet mass intervals (right) [23].

Further examples of simple di-object searches are for new heavy states decaying to dileptons: $e e, \mu \mu, e v$ or $\mu v$. ATLAS reported such searches and constraints, with limits on a sequential SM-like $Z^{\prime}$ for example being placed [24] $m(Z)>4.05 \mathrm{TeV}$ at $95 \% \mathrm{CL}$, compared to $2.90 \mathrm{TeV}$ from Run-1; and for a SM-like $W^{\prime}[25], m\left(W^{\prime}\right)>4.74 \mathrm{TeV}$ at $95 \% \mathrm{CL}$, compared to $3.24 \mathrm{TeV}$ from Run-1. Again, no evidence is seen in any of these channels for anomalous production not described by the Standard Model.
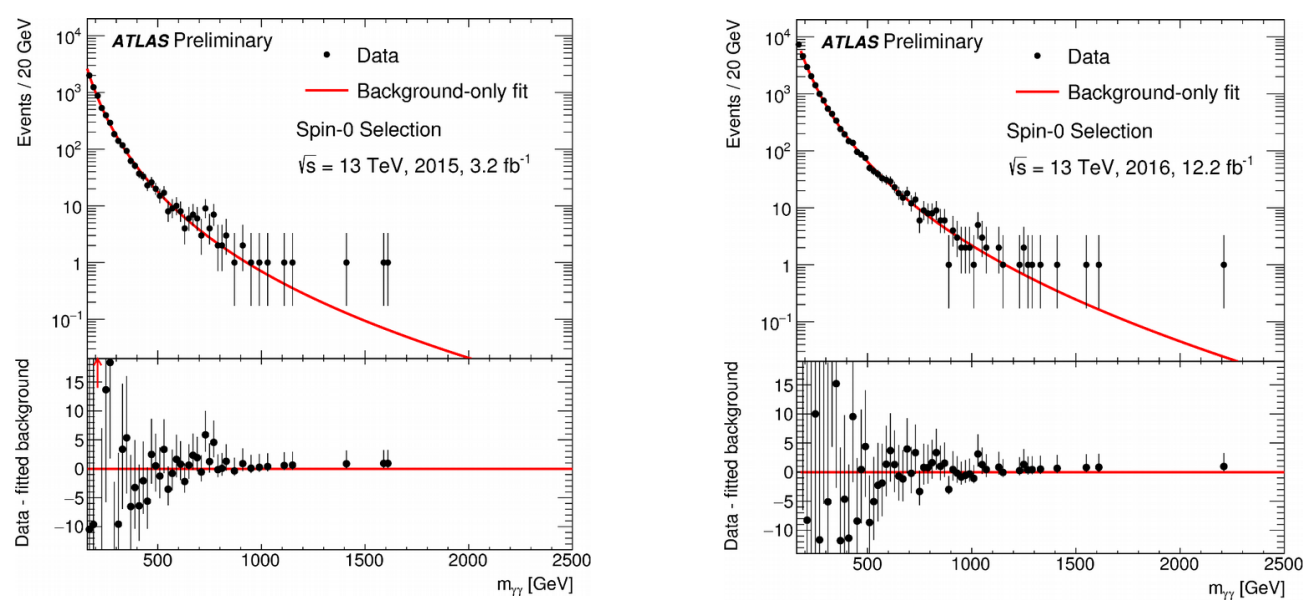

Figure 8: Diphoton invariant mass distributions in the high-mass scalar-optimised search in 2015 data (left) and 2016 data (right) [28].

For the diphoton final-state signature, some interest had arisen following results shown from the 2015 data alone, where a modest clustering of events $(2.1 \sigma$ global significance level, taking into account the look-elsewhere effect within this analysis) had been noted by ATLAS around $750 \mathrm{GeV}$ invariant mass [26], with support also from CMS data combined over 8 and 13 $\mathrm{TeV}$ collisions [27]. The behaviour in the four-times larger 2016 ATLAS data sample was reported. Results [28] were shown for the analysis optimised for spin-0 particle production: no 
clustering of events around $750 \mathrm{GeV}$ is observed in the 2016 data alone, as illustrated in Figure 8. Combining 2015 and 2016 data, the local significance of any excess around $750 \mathrm{GeV}$ is just $1.4 \sigma$.

A broad set of results were reported by ATLAS at the conference on searches for supersymmetric particle production, many of which may also be interpreted in more generic dark matter scenarios. Such analyses often adopt a common strategy: specific event signatures are sought, using simplified models with a single decay chain as a guideline for optimisation. Multiple complementary signal regions are often used, to cover a range of signal hypotheses (e.g. mass values of $\bar{q}, \bar{g}$ and lightest SUSY particle, LSP). Backgrounds are estimated using data-driven techniques, employing control regions close to the signal regions to constrain simulations of backgrounds, and often checking validation regions to test the data-driven approach closer to the signal region(s). Searches in complex final states are greatly assisted by the use of recent Monte Carlo programs, which provide better modelling than those used for Run-1 analyses, as is verified from the validation regions. Two example analyses reported here are: one part of the search for strong pair production of squarks or gluinos in $R$-parity conserving models; and searches for stop-pair production.

In the case of strong production of squarks and gluinos, the cross-sections would be rather high, compared for example to production of similar-mass particles produced only via electroweak processes: this results in strongly enhanced sensitivity, compared to Run-1, already from the data sample available at the time of the conference. One analysis [29] selects events with 2-6 jets and missing transverse momentum $\left(E_{\mathrm{T}}{ }^{\text {miss }}\right)$, vetoing events with identified leptons. As many as 30 signal regions are defined using jet multiplicity and the reconstructed $m_{\text {eff }}$ variable, defined as $m_{\text {eff }}=E_{\mathrm{T}}^{\text {miss }}+\Sigma \mid p_{\mathrm{T}}($ jet $) \mid$, for example. An example of the sensitivity at high $m_{\text {eff }}$ is ilustrated in Figure 9, together with limits derived for a simplified model of gluino pair production with gluino decaying to $q \bar{q} \bar{\chi}_{1}^{0}$, where the $\widetilde{\chi}_{1}^{0}$ is the LSP. A very striking improvement in sensitivity beyond Run-1 is clear. With the new data sample, in this simplified model, a $95 \%$ CL lower mass limit of $1.86 \mathrm{TeV}$ is obtained, for the case of a low-mass $\tilde{\chi}_{1}^{0}$.
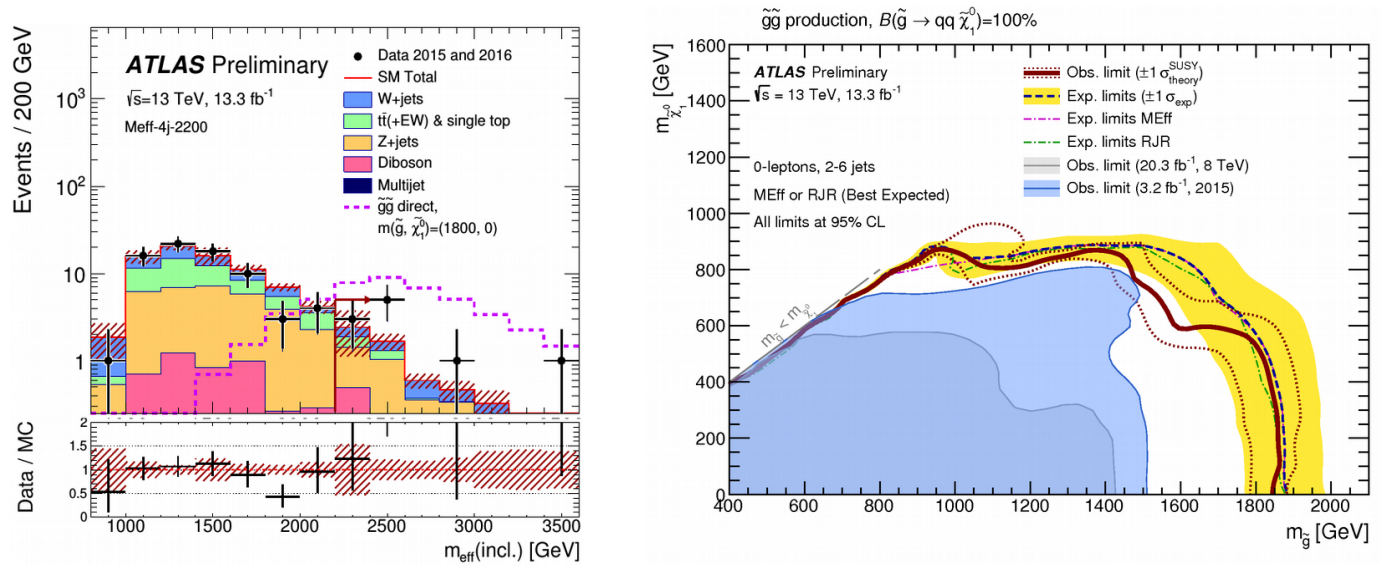

Figure 9: (Left) Distribution of $m_{\text {eff }}$ for one signal region of the no-lepton, 2-6 jets strong SUSY search [29], with the $m_{\text {eff }}$ selection released and indicated; (right) limits in the $\bar{\chi}_{1}^{0}$ vs $\bar{g}$ mass plane in the simplified model with $100 \%$ of $g$ decaying to $q \overline{q \chi}_{1}^{0}[29]$. 
In the stop searches, new analysis techniques are used which improve sensitivity to areas of SUSY mass-space which have not previously been well-probed, specifically for compressed scenarios where the stop mass is close to the sum of the masses of its decay products, so there is little $E_{\mathrm{T}}{ }^{\text {miss }}$ in the stop rest frame. Since there is typically still a massive LSP and thus large missing mass, in configurations with boosted stops the $E_{\mathrm{T}}$ miss can still be significant in the lab frame. These techniques allow substantial improvements in sensitivity. No significant excesses are seen in the data [30], and this can be seen in the extended limits obtained with Run-2 data compared to Run-1, as shown for example in Figure 10 - note the improved exclusions in the "diagonal" regions with compressed mass configurations.

Figure 10: Exclusions from various updated analyses in the $\bar{\chi}_{1}^{0}$ vs $\tilde{t}$ mass plane; in each analysis the specified simplified models are considered.

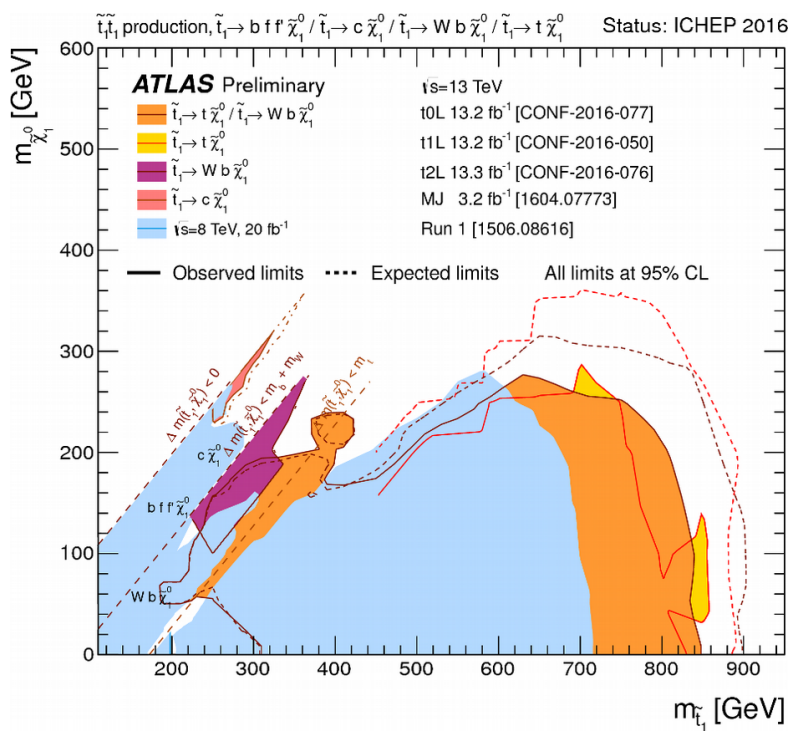

An important set of new physics probes comes by studying massive diboson production, either pairs of electroweak vector bosons, or a $W$ or $Z$ produced together with an $H(125)$, or searching for di-Higgs production. ATLAS presented results on many such channels at the conference, including the 2016 data. Hadronic decays of the $W$ and $Z$ can be probed using boosted object techniques, to extend into regions where the jets from a single boson decay are merged into a "fat jet". In-situ validations of boosted object performance have been carried out [31]. In all of these searches [31, 32], again no significant evidence of non-Standard Model production has been found. An example summary plot is shown in Figure 11, of the different searches conducted for high-mass anomalous $W Z$ production from a $W^{\prime}$ decay. Upper limits are placed on cross-sections times branching fractions to $W Z$, for $W^{\prime}$ masses above $2 \mathrm{TeV}$, at better than $10 \mathrm{fb}$.

Figure 11: Upper limits on crosssections for production of a new $\mathrm{W}^{\prime}$ state decaying to the $\mathrm{WZ}$, as derived from searches in the decay modes indicated.

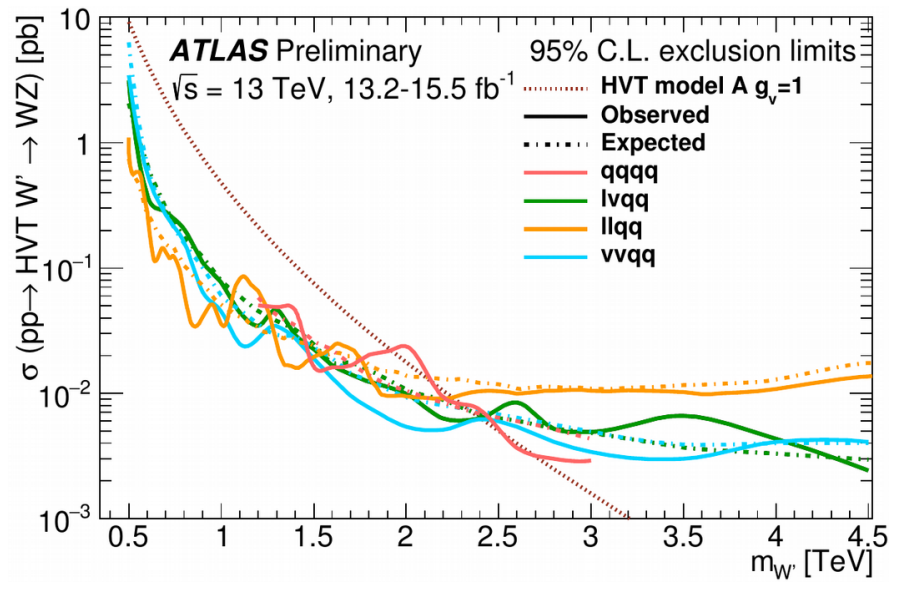




\section{Summary and Conclusion}

In summary, a wealth of results have been reported by ATLAS and LHCb at this conference. The performance of the LHC in 2016 is extraordinary, and that of the detectors in Run-2 is also excellent, despite high pileup levels well beyond the design levels. LHCb has shown results including a $b \bar{b}$ cross-section measurement at $13 \mathrm{TeV}$, and $\mathrm{CP}$ violation studies in the baryon and charm sectors, amongst many others. ATLAS has prepared more than 60 new results for this conference, including a spectrum of measurements using $13 \mathrm{TeV}$ collision data. In the scalar sector of the Standard Model, the $H(125)$ is clearly re-established in $13 \mathrm{TeV}$ collisions, and in-depth studies are ongoing aimed at establishing $t t H$ production and the $H \rightarrow b b$ decay with Run-2 data. A large effort has been put into investigating a wide range of search topologies using the 2015 and 2016 data, providing a broad exploration of many areas, with sensitivity well beyond Run-1. The timing of this conference means that the search sensitivity in ATLAS was substantially improved over the 2015 data, and Run-1, in many channels: a large potential space for new physics opened up - a bigger step in sensitivity in a few months than will be available for many years to come, although data continue to pour in. While there are a few modest excesses at the 2-3 $\sigma$ level, these are generally at the level expected from background fluctuations, and are not significant enough to stress here. The overall message is that the Standard Model, using the latest generations of Monte Carlo generators and calculations, continues to describe the data with astonishing fidelity.

\section{Acknowledgements}

The results presented are the responsibility of, and shown on behalf of, the ATLAS and LHCb Collaborations, which were built, and operate, thanks to the support of a range of funding agencies across the globe, too numerous to list here, as well as depending sine qua non on the world-wide LHC computing grid. The speaker acknowledges the specific help in the preparation of this contribution from B. Heinemann, M. Kado and V. Vagnoni.

\section{References}

[1] ATLAS Collaboration, Observation of a new particle in the search for the Standard Model Higgs boson with the ATLAS detector at the LHC, Phys. Lett. B716 (2012) 1-29 [arxiv:1207.7214]; CMS Collaboration, Observation of a new boson at a mass of $125 \mathrm{GeV}$ with the CMS experiment at the LHC, Phys. Lett. B716 (2012) 30-61 [arXiv:1207.7235].

[2] M. Lamont, LHC accelerator performance, this conference.

[3] LHCb Collaboration, The LHCb detector at the LHC, JINST 3 (2008) S08005.

[4] ATLAS Collaboration, The ATLAS Experiment at the CERN Large Hadron Collider, JINST 3 (2008) S08003.

[5] ATLAS Collaboration, Performance of the ATLAS Trigger system in 2015, arXiv:1611.09661. 
[6] LHCb Collaboration, Measurement of the b-quark production cross-section in 7 and $13 \mathrm{TeV} p p$ collisions, Phys. Rev. Lett. 118 (2017) 052002 [arXiv:1612.05140]; this result and plot are updated to the paper version from that shown at the conference.

[7] ATLAS Collaboration, Measurement of the tt production cross-section using e $\mu$ events with $b$ tagged jets in pp collisions at $\sqrt{s}_{s}=13 \mathrm{TeV}$ with the ATLAS detector, Phys. Lett. B761 (2016) 136-157 [arXiv:1606.02699]

[8] M. Cacciari, M. L. Mangano, and P. Nason, Gluon PDF constraints from the ratio of forward heavyquark production at the LHC at $\sqrt{s}_{\mathrm{s}}=7$ and $13 \mathrm{TeV}$, Eur. Phys. J. C 75, 610 (2015) [arXiv:1507.06197].

[9] ATLAS Collaboration, Measurements of top-quark pair differential cross-sections in the lepton+jets channel in pp collisions at $\sqrt{s}_{s}=13$ TeV using the ATLAS detector, ATLAS-CONF-2016-040, August 2016 (unpublished).

[10] ATLAS Collaboration, Measurements of the Production Cross Section of a Z boson in Association with Jets in pp collisions at $\sqrt{ }_{s}=13 \mathrm{TeV}$ with the ATLAS Detector, ATLAS-CONF-2016-046, August 2016 (unpublished).

[11] ATLAS Collaboration, Measurement of the $W^{ \pm} Z$ boson pair-production cross section in pp collisions at $\sqrt{ }_{s}=13 \mathrm{TeV}$ with the ATLAS Detector, Phys. Lett. B 762 (2016) 1 [arXiv:1606.04017].

[12] M. Grazzini, S. Kallweit, D. Rathlev and M. Wiesemann, $W^{ \pm} Z$ production at hadron colliders in NNLO QCD, (2016), Phys. Lett. B761 (2016) 179-183 [arXiv:1604.08576].

[13] LHCb Collaboration, Measurement of matter-antimatter differences in beauty baryon decays, to appear in Nature Phys. [arXiv:1609.05216].

[14] LHCb Collaboration, Measurement of CP asymmetry in $D^{0} \rightarrow K^{-} K^{+}$decays, Phys. Lett. B 767 (2017) 177-187 [arXiv:1610.09476].

[15] V. Vagnoni, Results and prospects on bottom physics, this conference.

[16] ATLAS Collaboration, Measurement of fiducial, differential and production cross sections in the $H \rightarrow \gamma \gamma$ decay channel with $13.3 \mathrm{fb}^{-1}$ of $13 \mathrm{TeV}$ proton-proton collision data with the ATLAS detector, ATLAS-CONF-2016-067, August 2016 (unpublished).

[17] ATLAS Collaboration, Study of the Higgs boson properties and search for high-mass scalar resonances in the $H \rightarrow Z Z * \rightarrow 4 \ell$ decay channel at $\sqrt{s}=13$ TeV with the ATLAS detector, ATLASCONF-2016-079, August 2016 (unpublished).

[18] ATLAS Collaboration, Combined measurements of the Higgs boson production and decay rates in $H \rightarrow Z Z^{*} \rightarrow 4 \ell$ and $H \rightarrow \gamma \gamma$ final states using $p p$ collision data at $\sqrt{s}_{s}=13 \mathrm{TeV}$ in the ATLAS experiment, ATLAS-CONF-2016-081, August 2016 (unpublished).

[19] LHC Higgs Cross Section Working Group, D. de Florian et al., Handbook of LHC Higgs Cross Sections: 4. Deciphering the Nature of the Higgs Sector, arXiv:1610.07922.

[20] ATLAS Collaboration, Search for the Associated Production of a Higgs Boson and a Top Quark Pair in Multilepton Final States with the ATLAS Detector, ATLAS-CONF-2016-058, August 2016 (unpublished); ATLAS Collaboration, Search for the Standard Model Higgs boson produced in association with top quarks and decaying into a bb pair in pp collisions at $\sqrt{s}_{\mathrm{s}}=13 \mathrm{TeV}$ with the ATLAS detector, ATLAS-CONF-2016-080, August 2016 (unpublished).

[21] ATLAS Collaboration, Search for the Standard Model Higgs boson produced in association with a vector boson and decaying to a b $\bar{b}$ pair in pp collisions at $13 \mathrm{TeV}$ using the ATLAS detector, ATLAS-CONF-2016-091, August 2016 (unpublished). 
[22] See, for example, W. Adam, Searches for Supersymmetry; and S-C. Hsu, Exotica searches, this conference.

[23] ATLAS Collaboration, Search for new phenomena in dijet events collected in 2015 and 2016 pp collisions with the ATLAS detector at $\sqrt{s}_{s}=13 \mathrm{TeV}$, ATLAS-CONF-2016-069, August 2016 (unpublished).

[24] ATLAS Collaboration, Search for new high-mass resonances in the dilepton final state using proton--proton collisions at $\sqrt{ }_{s}=13 \mathrm{TeV}$ with the ATLAS detector, ATLAS-CONF-2016-045, August 2016 (unpublished).

[25] ATLAS Collaboration, Search for new resonances decaying to a charged lepton and a neutrino in pp collisions at $\sqrt{s}_{s}=13 \mathrm{TeV}$ with the ATLAS detector, ATLAS-CONF-2016-061, August 2016 (unpublished).

[26] ATLAS Collaboration, Search for resonances in diphoton events at $\sqrt{s}_{s}=13 \mathrm{TeV}$ with the ATLAS detector, JHEP 09 (2016) 1 [arXiv:1606.03833]

[27] CMS Collaboration, Search for resonant production of high-mass photon pairs in proton-proton collisions at $\sqrt{s}_{s}=8$ and $13 \mathrm{TeV}$, Phys. Rev. Lett. 117 (2016) 051802 [arXiv:1606.04093].

[28] ATLAS Collaboration, Search for scalar diphoton resonances with $15.4 \mathrm{fb}^{-1}$ of data collected at $\sqrt{s}_{s}=13 \mathrm{TeV}$ in 2015 and 2016 with the ATLAS detector, ATLAS-CONF-2016-059, August 2016 (unpublished).

[29] ATLAS Collaboration, Further searches for squarks and gluinos in final states with jets and missing transverse momentum at $\sqrt{s}_{\mathrm{s}}=13 \mathrm{TeV}$ with the ATLAS detector, ATLAS-CONF-2016-078, August 2016 (unpublished).

[30] See for example: ATLAS Collaboration, Search for top squarks in final states with one isolated lepton, jets, and missing transverse momentum in $\sqrt{s}_{s}=13 \mathrm{TeV}$ pp collisions with the ATLAS detector, ATLAS-CONF-2016-050, August 2016 (unpublished); ATLAS Collaboration, Search for direct top squark pair production and Dark Matter production in final states with two leptons in $\sqrt{s}_{s}=13 \mathrm{TeV}$ pp collisions using $13 \mathrm{fb}^{-1}$ of ATLAS data, ATLAS-CONF-2016-076, August 2016 (unpublished); ATLAS Collaboration, Search for a Scalar Partner of the Top Quark in the Jets $+E_{T}^{\text {miss }}$ Final State at $\sqrt{s}_{s}=13 \mathrm{TeV}$ with the ATLAS detector, ATLAS-CONF-2016-077, August 2016 (unpublished).

[31] ATLAS Collaboration, Search for resonances with boson-tagged jets in $15.5 \mathrm{fb}^{-1}$ of pp collisions at $\sqrt{ }_{s}=13 \mathrm{TeV}$ collected with the ATLAS detector, ATLAS-CONF-2016-055, August 2016 (unpublished).

[32] ATLAS Collaboration, Search for pair production of Higgs bosons in the $b \bar{b} b \bar{b}$ final state using proton-proton collisions at $\sqrt{s}_{s}=13 \mathrm{TeV}$ with the ATLAS detector, ATLAS-CONF-2016-049, August 2016 (unpublished); ATLAS Collaboration, Searches for heavy ZZ and ZW resonances in the $\ell \ell q q$ and vvqq final states in pp collisions at $\sqrt{S}_{S}=13 \mathrm{TeV}$ with the ATLAS detector, ATLAS-CONF-2016082, August 2016 (unpublished); ATLAS Collaboration, A Search for Resonances Decaying to a $W W$ or ZZ Boson and $a$ Higgs Boson in the $q \bar{q}\left({ }^{\prime}\right) b \bar{b}$ Final State, ATLAS-CONF-2016-083, August 2016 (unpublished). 